TITLE:

\title{
Generalization of an isotropic vector hysteresis model represented by the superposition of stop models - Identification and rotational hysteresis loss
}

\author{
AUTHOR(S): \\ Matsuo, T; Shimasaki, M
}

\section{CITATION:}

Matsuo, T ... [et al]. Generalization of an isotropic vector hysteresis model represented by the superposition of stop models - Identification and rotational hysteresis loss. IEEE TRANSACTIONS ON MAGNETICS 2007, 43(4): 1389-1392

\section{ISSUE DATE:}

2007-04

URL:

http://hdl.handle.net/2433/50129

\section{RIGHT:}

(c)2007 IEEE. Personal use of this material is permitted. However, permission to reprint/republish this material for advertising or promotional purposes or for creating new collective works for resale or redistribution to servers or lists, or to reuse any copyrighted component of this work in other works must be obtained from the IEEE. 


\title{
Generalization of an Isotropic Vector Hysteresis Model Represented by the Superposition of Stop Models-Identification and Rotational Hysteresis Loss
}

\author{
T. Matsuo and M. Shimasaki \\ Graduate School of Engineering, Kyoto University, Kyoto 615-8510, Japan
}

\begin{abstract}
This paper generalizes a 2-D isotropic vector hysteresis model represented by the superposition of scalar stop models. Its identification method and property of rotational hysteresis loss are discussed. The generalized vector model can control its rotational hysteresis loss.
\end{abstract}

Index Terms-Identification, rotational hysteresis loss, superposition, vector stop model.

\section{INTRODUCTION}

A CCURATE and detailed computation of magnetic fields and power losses in electrical machines remains difficult because of complex magnetic properties of iron-core materials, including hysteresis and vector properties. A precise and efficient vector hysteresis model is therefore required for accurate and efficient magnetic-field analyses in iron cores.

Several vector hysteresis models have been proposed, such as vector versions of the Preisach model [1], [2]; the Stoner-Wohlfarth model [3]; and the E \& SS model [4]. For example, Mayergoyz proposed a 2-D vector Preisach model [1], which is constructed using a superposition of scalar Preisach models along the angular direction. To improve its rotational representation, Mayergoyz also proposed a generalized version of this vector model and its identification methods [1].

The Preisach model has been widely used to describe complex hysteretic characteristics precisely. However, large-scale electromagnetic-field analyses of iron-core materials require a more efficient hysteresis model because the Preisach model has high computational costs, such as a large memory requirement and the calculation cost to obtain a magnetic field $H$ from a magnetic flux density $B$ [5].

The stop model [6]-[8] is one alternative hysteresis model to the Preisach model because of its efficiency. It can directly provide a hysteretic output of $H$ from an input $B$, whereas the Preisach model usually obtains an output $B$ (or a magnetization $M$ ) from $H$. Moreover, the stop model can be simply implemented with a small memory requirement compared with the Preisach model.

Several vector versions of the stop model have been proposed, such as geometric extensions of the scalar model [6], [9] and a superposition of scalar models [10], [11]. The latter is constructed in the same way as the vector Preisach model by Mayergoyz [1]; it

Digital Object Identifier 10.1109/TMAG.2007.892427

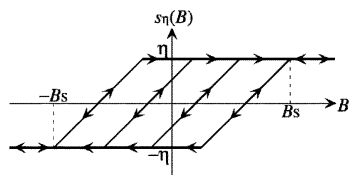

Fig. 1. Stop hysteron operator given by (2) and (3).

has been applied to a 2-D eddy current analysis [10]. However, its rotational hysteretic property has not been discussed.

The present study generalizes the vector stop model to improve its rotational hysteretic property. Its identification method and property of rotational hysteresis loss are discussed.

\section{SCALAR STOP MODELS}

\section{A. Stop Model With Input-Independent Shape Function}

The stop model with an input-independent shape function gives a hysteretic relation between an input $B$ and an output $H$ as

$$
H=S(B)=\int_{0}^{B \mathrm{~s}} g\left(\eta, s_{\eta}(B)\right) \mathrm{d} \eta
$$

where $B_{\mathrm{S}}$ is the saturation magnetic flux density and $g$ is an input-independent shape function. This paper defines the stop hysteron $s_{\eta}$ having a height $\eta$ as the following:

$$
\begin{aligned}
S_{\eta}(B) & = \begin{cases}\max \left(\min \left(B-B^{0 *}+\mathrm{S}_{\eta}^{0}, \eta\right),-\eta\right) & \left(\eta<B_{S}\right) \\
B & \left(\eta=B_{S}\right)\end{cases} \\
B^{0 *} & =\max \left(\min \left(B^{0}, B_{\mathrm{S}}\right),-B_{\mathrm{S}}\right) .
\end{aligned}
$$

Therein, $B^{0}$ and $s_{\eta}^{0}$ are values of $B$ and $s_{\eta}$ at the previous timepoint. The variable $B_{0}^{*}$ is introduced so that $s_{\eta}(B)$ does not exhibit a hysteretic property when $|B| \geq B_{\mathrm{S}}$. The hysteron $s_{\eta}(B)$ becomes a single-valued function when $\eta=B_{\mathrm{S}}$. Fig. 1 illustrates the characteristics of this operator for $\eta<B_{\mathrm{S}}$.

A hysteretic function is generally decomposed into an irreversible component and a reversible component [2]. The reversible component of the stop model (1) is given as

$$
G(B)=\lim _{\delta \rightarrow+0} \int_{B \mathrm{~s}-\delta}^{B \mathrm{~s}} g\left(\eta, s_{\eta}(B)\right) \mathrm{d} \eta .
$$


If the reversible part (4) is nonzero, the shape function $g(\eta, s)$ becomes singular at $\eta=B_{\mathrm{S}}$. To avoid singularity, the stop model (1) is decomposed into an irreversible component $(\eta<$ $\left.B_{\mathrm{S}}\right)$ and a reversible component $\left(\zeta=B_{\mathrm{S}}\right)[11]$ as

$$
S(B)=G(B)+\int_{0}^{B \mathrm{~s}-0} g\left(\eta, s_{\eta}(B)\right) \mathrm{d} \eta .
$$

\section{B. Hysteresis Loss}

For an alternating input of $B$ with amplitude $B_{\mathrm{a}}$, the stop model $S(B)$ yields a hysteresis loss $L_{\text {alt }}\left(B_{\mathrm{a}}\right)$ per cycle as

$L_{\text {alt }}\left(B_{\mathrm{a}}\right)=\oint_{B \mathrm{a}} S(B) \mathrm{d} B=\int_{0}^{B_{\mathrm{S}}-0} \oint_{B \mathrm{a}} g\left(\eta, s_{\eta}(B)\right) \mathrm{d} B \mathrm{~d} \eta$.

When $B_{\mathrm{a}}>\eta, B$ and $g\left(\eta, s_{\eta}(B)\right)$ make a hysteresis loop as shown in Fig. 2. its loop area is given as

$$
\oint_{B \mathrm{a}} g\left(\eta, s_{\eta}(B)\right) \mathrm{d} B= \begin{cases}4\left(B_{\mathrm{a}}-\eta\right) g(\eta, \eta) & \left(B_{\mathrm{a}}<B_{\mathrm{S}}\right) \\ 4\left(B_{\mathrm{S}}-\eta\right) g(\eta, \eta) & \left(B_{\mathrm{a}} \geq B_{\mathrm{S}}\right)\end{cases}
$$

where $g(\eta,-\eta)=-g(\eta, \eta)$ is assumed. Consequently, the alternating hysteresis loss $L_{\text {alt }}\left(B_{\mathrm{a}}\right)$ is given as

$$
\begin{gathered}
L_{\text {alt }}\left(B_{\mathrm{a}}\right)=4 \int_{0}^{B \mathrm{~m}}\left(B_{\mathrm{m}}-\eta\right) g(\eta, \eta) \mathrm{d} \eta \\
\text { where } r B_{\mathrm{m}}=\min \left(B_{\mathrm{a}}, B_{\mathrm{S}}\right) .
\end{gathered}
$$

Equation (8) implies that the hysteresis loss becomes constant for $B_{\mathrm{a}} \geq B_{\mathrm{S}}$.

\section{Stop Model With Input-Dependent Shape Function}

An input-dependent shape function has been proposed to improve the representation capability of the stop model [12]. The stop model with an input-dependent shape function is given as

$$
S_{\mathrm{w}}(B)=\int_{0}^{B \mathrm{~s}} g_{\mathrm{w}}\left(\eta, s_{\eta}(B), B\right) \mathrm{d} \eta
$$

where $g_{\mathrm{w}}$ is an input-dependent shape function. The stop model (10) has been proven equivalent [13] to the nonlinear Preisach model proposed by Mayergoyz [1].

A product form of the input-dependent shape function $g_{\mathrm{w}}(\eta, s, B)=g(\eta, s) w(B)$ was introduced in [12], where $w(B)$ is called a weighting function. This product form gives the stop model as

$$
S_{\mathrm{w}}(B)=w(B) \int_{0}^{B \mathrm{~s}} g\left(\eta, s_{\eta}(B)\right) \mathrm{d} \eta .
$$

\section{VECTOR STOP MODELS}

\section{A. Vector Model by the Superposition of Scalar Models}

Similar to the vector Preisach model proposed by Mayergoyz [1], a 2-D isotropic vector stop model (1) is constructed through the superposition of scalar stop models [11]

$$
\boldsymbol{H}=\boldsymbol{S}(\boldsymbol{B})=\int_{-\pi / 2}^{\pi / 2} \boldsymbol{e}_{\varphi} S_{2}\left(\boldsymbol{e}_{\varphi} \cdot \boldsymbol{B}\right) \mathrm{d} \varphi
$$

Therein, $\boldsymbol{e}_{\varphi}$ is the unit vector along the $\varphi$-direction and $S_{2}(B)$ is a scalar stop model (1). An identification method of this vector stop model is given in [11].

When a rotational input

$$
\boldsymbol{B}=\left(B_{\mathrm{a}} \cos \omega t, B_{\mathrm{a}} \sin \omega t\right)
$$
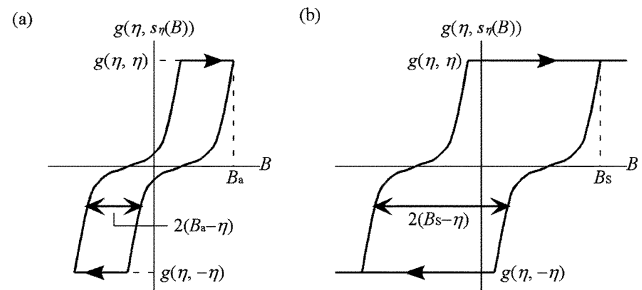

Fig. 2. Hysteresis loop of $B-g\left(\eta, s_{\eta}(B)\right)$ for $B_{\text {a }}>\eta$. (a) $B<B_{S}$. (b) $B \geq B_{S}$

is given, the vector model $\boldsymbol{S}(\boldsymbol{B})$ yields a hysteresis loss

$L_{\mathrm{rot}}\left(B_{\mathrm{a}}\right)$ as

$L_{\mathrm{rot}}\left(B_{\mathrm{a}}\right)=\oint_{B \mathrm{a}} \boldsymbol{S}(\boldsymbol{B}) \cdot \mathrm{d} \boldsymbol{B}$
$=\int_{-\pi / 2}^{\pi / 2} \int_{0}^{2 \pi}-S_{2}\left(B_{\mathrm{a}} \cos (\omega t-\varphi)\right) B_{\mathrm{a}} \sin (\omega t-\varphi) \mathrm{d}(\omega t) \mathrm{d} \varphi$
$=\int_{-\pi / 2}^{\pi / 2} \oint_{B \mathrm{a}} S_{2}(B) \mathrm{d} B \mathrm{~d} \varphi=\pi \oint_{B \mathrm{a}} S_{2}(B) \mathrm{d} B=\pi L_{2 \mathrm{alt}}\left(B_{\mathrm{a}}\right)$.

Therein, $L_{2 \text { alt }}$ is the hysteresis loss given by the scalar model $S_{2}(B)$ in the same way as (8). Since $L_{2 \text { alt }}\left(B_{\mathrm{a}}\right)$ is constant for $B_{\mathrm{a}} \geq B_{\mathrm{S}}, L_{\mathrm{rot}}\left(B_{\mathrm{a}}\right)$ is also constant for $B_{\mathrm{a}} \geq B_{\mathrm{S}}$. This constant rotational hysteresis loss is not observed in actual ferromagnetic materials.

On the other hand, when a unidirectional alternating input with amplitude $B_{\mathrm{a}}$ is given, $\boldsymbol{S}(\boldsymbol{B})$ yields a hysteresis loss $L_{\text {alt }}\left(B_{\mathrm{a}}\right)$ as

$$
\begin{aligned}
L_{\mathrm{alt}}\left(B_{\mathrm{a}}\right) & =\int_{-\pi / 2}^{\pi / 2} \oint_{B \mathrm{a}} S_{2}(B \cos \varphi) \cos \varphi \mathrm{d} B \mathrm{~d} \varphi \\
& =\int_{-\pi / 2}^{\pi / 2} L_{2 \mathrm{alt}}\left(B_{\mathrm{a}} \cos \varphi\right) \mathrm{d} \varphi \\
& =\frac{2}{\pi} \int_{0}^{\pi / 2} L_{\mathrm{rot}}\left(B_{\mathrm{a}} \cos \varphi\right) \mathrm{d} \varphi .
\end{aligned}
$$

\section{B. Generalized Vector Model}

Similar to the generalized vector Preisach model proposed by Mayergoyz [1], the vector stop model (12) is generalized as

$$
\boldsymbol{H}=\boldsymbol{S}(\boldsymbol{B})=\int_{-\pi / 2}^{\pi / 2} \boldsymbol{e}_{\varphi} S_{2}\left(\|\boldsymbol{B}\| \cos ^{1 / n}(\theta-\varphi)\right) \mathrm{d} \varphi .
$$

Therein, $\theta$ is the angle of $\boldsymbol{B}$, and $n$ is a projection parameter for input $\boldsymbol{B}$ to the $\varphi$-direction; $\cos ^{1 / n} \phi$ denotes the $|\cos \phi|^{1 / n}$ sign $(\cos \phi)$ for the simplicity of expression. When $n=1$, vector model (16) coincides with the original model (12).

For a rotational input (13), the vector model (16) yields a hysteresis loss $L_{\text {rot }}\left(B_{\mathrm{a}}\right)$ as

$$
\begin{aligned}
L_{\text {rot }} & \left(B_{\mathrm{a}}\right)=\int_{-\pi / 2}^{\pi / 2} \int_{0}^{2 \pi}-S_{2}\left(B_{\mathrm{a}} \cos ^{1 / n}(\omega t-\varphi)\right) \\
& \times B_{\mathrm{a}} \sin (\omega t-\varphi) \mathrm{d}(\omega t) \mathrm{d} \varphi \\
= & \int_{-\pi / 2}^{\pi / 2} \oint_{B \mathrm{a}} S_{2}\left(B_{\mathrm{a}}\left(\frac{B}{B_{\mathrm{a}}}\right)^{1 / n}\right) \mathrm{d} B \mathrm{~d} \varphi \\
= & \pi \oint_{B \mathrm{a}} S_{2}\left(B_{\mathrm{a}}\left(\frac{B}{B_{\mathrm{a}}}\right)^{1 / n}\right) \mathrm{d} B
\end{aligned}
$$



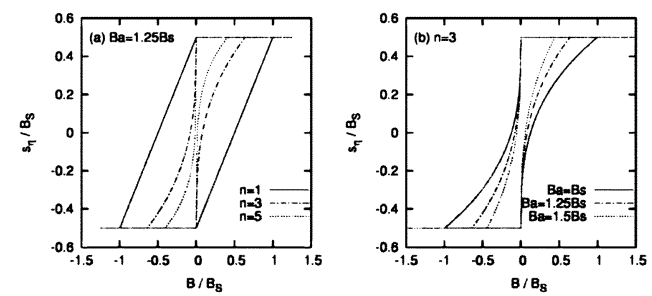

Fig. 3. Hysteresis loops of $B-s_{\eta}\left(B_{\mathrm{a}}\left(B / B_{\mathrm{a}}\right)^{1 / n}\right)$ when $\eta=0.5 B_{\mathrm{S}}$ and $B_{\mathrm{a}} \geq B_{\mathrm{S}}$

For input $B_{\mathrm{a}}\left(B / B_{\mathrm{a}}\right)^{1 / n}$, the stop hystron $s_{\eta}$ responds as

$s_{\eta}\left(B_{\mathrm{a}}\left(\frac{B}{B_{\mathrm{a}}}\right)^{\frac{1}{n}}\right)=$

$\left\{\begin{array}{l}\max \left(\min \left(B_{\mathrm{a}}\left(\frac{B}{B_{\mathrm{a}}}\right)^{\frac{1}{n}}-\eta+B_{\mathrm{m}}, \eta\right),-\eta\right)(\text { when increasing) } \\ \max \left(\min \left(B_{\mathrm{a}}\left(\frac{B}{B_{\mathrm{a}}}\right)^{\frac{1}{n}}+\eta-B_{\mathrm{m}}, \eta\right),-\eta\right) \text { (when decreasing). }\end{array}\right.$

Fig. 3 illustrates the relation (18) for $B_{\mathrm{a}} \geq B_{\mathrm{S}}$. For rotational input (13), the width of the hysteresis loop decreases with the increase in $n$ and $B_{\mathrm{a}}$. Accordingly, the rotational loss $L_{\mathrm{rot}}(B)$ decreases with the increase in amplitude $B_{\mathrm{a}}$ when $B_{\mathrm{a}}>B_{\mathrm{S}}$ and $n>1$.

\section{Identification of the Generalized Vector Model}

The vector stop model (16) is identified from its unidirectional property. The unidirectional property along $\theta=0$ is given as

$$
H=S_{1}(B)=\int_{-\pi / 2}^{\pi / 2} S_{2}\left(B \cos ^{1 / n} \varphi\right) \cos \varphi \mathrm{d} \varphi
$$

where $S_{1}$ is a scalar hysteresis property represented by a scalar stop model. Several identification methods [8], [11], [12] have been proposed to determine the shape function of the scalar stop model $S_{1}$ from measured unidirectional properties. The stop model $S_{2}$ is identified from $S_{1}$ as follows.

For identification, the following function $T_{k}$ is defined:

$$
\begin{aligned}
& T_{k}(\eta, s) \\
& = \begin{cases}G_{k}(s)+\int_{\eta}^{B s-0} g k(\xi, s) \mathrm{d} \xi & \left(\eta<B_{\mathrm{S}}\right) \\
G_{k}(s) & \left(\eta=B_{\mathrm{S}}\right) .\end{cases}
\end{aligned}
$$

In that definition, $G_{k}$ and $g_{k}(k=1,2)$, respectively, denote the reversible component and the shape function of $S_{k}$. Relation (19) requires a relation (21) between $T_{1}$ and $T_{2}$.

$$
T_{1}(\eta, s)=\int_{-\pi / 2}^{\pi / 2} T_{2}\left(\eta \cos ^{1 / n} \varphi, s \cos ^{1 / n} \varphi\right) \cos \varphi \mathrm{d} \varphi
$$

This integral equation is solved for $T_{2}$ as (22) and (23)

$$
\begin{aligned}
T_{2}(\eta, s) & =\frac{1}{\pi} \int_{0}^{\pi / 2} T_{12}\left(\eta \cos ^{1 / n} \varphi, s \cos ^{1 / n} \varphi\right) \mathrm{d} \varphi \\
T_{12}(\eta, s) & =T_{1}(\eta, s)+\frac{\eta}{n} \frac{\partial T_{1}(\eta, s)}{\partial \eta}+\frac{s}{n} \frac{\partial T_{1}(\eta, s)}{\partial s}
\end{aligned}
$$

Functions $g_{2}$ and $G_{2}$ are given from $T_{2}$ by (24)

$$
G_{2}(s)=T_{2}\left(B_{\mathrm{S}}, s\right), g_{2}(\eta, s)=-\frac{\partial T_{2}(\eta, s)}{\partial \eta}\left(\eta<B_{\mathrm{S}}\right) .
$$

\section{Generalized Vector Model With Weighting Function}

Similar to the scalar stop model (11), the vector stop model is further generalized as (25)

$$
\begin{aligned}
\boldsymbol{H}=\boldsymbol{S}_{\mathrm{w}}(\boldsymbol{B})= & w(\|\boldsymbol{B}\|) \\
& \times \int_{-\pi / 2}^{\pi / 2} \boldsymbol{e}_{\varphi} S_{2}\left(\|\boldsymbol{B}\| \cos ^{1 / n}(\theta-\varphi)\right) \mathrm{d} \varphi .
\end{aligned}
$$

Equations (16) and (25) imply that $\boldsymbol{S}_{\mathrm{w}}(\boldsymbol{B}) / w(\|\boldsymbol{B}\|)=\boldsymbol{S}(\boldsymbol{B})$ is the generalized vector stop model (16).

The unidirectional property of $\boldsymbol{S}_{\mathrm{w}}(\boldsymbol{B})$ along $\theta=0$ is given as

$$
\begin{aligned}
H & =S_{\mathrm{w} 1}(B)=w(B) S_{1}(B) \\
& =w(B) \int_{-\pi / 2}^{\pi / 2} S_{2}\left(B \cos ^{1 / n} \varphi\right) \cos \varphi \mathrm{d} \varphi
\end{aligned}
$$

where $S_{\mathrm{w} 1}(B)$ is the scalar stop model (11). Several kinds of weighting functions [12]-[14] have been proposed to improve the representation of the scalar stop model. Stop model $S_{2}$ is identified by the method proposed in the previous subsection.

The rotational hysteresis loss $L_{\mathrm{wrot}}$ given by $\boldsymbol{S}_{\mathrm{w}}(\boldsymbol{B})$ for input (13) is

$$
L_{\mathrm{wrot}}\left(B_{\mathrm{a}}\right)=w\left(B_{\mathrm{a}}\right) L_{\mathrm{rot}}\left(B_{\mathrm{a}}\right)
$$

where $L_{\mathrm{rot}}$ is given by (17). When $n=1, L_{\mathrm{wrot}}$ becomes

$$
\begin{aligned}
L_{\text {wrot }}\left(B_{\mathrm{a}}\right) & =4 \pi w\left(B_{\mathrm{a}}\right) \int_{0}^{B \mathrm{~m}}\left(B_{\mathrm{m}}-\eta\right) g_{2}(\eta, \eta) \mathrm{d} \eta \\
& =\pi w\left(B_{\mathrm{a}}\right) L_{2 \text { alt }}\left(B_{\mathrm{a}}\right) .
\end{aligned}
$$

The rotational hysteresis loss depends on $w\left(B_{\mathrm{a}}\right)$. The scalar model $S_{2}$ and its alternating hysteresis loss $L_{2 a l t}$ also depend on the choice of $w(B)$. Accordingly, $L_{\mathrm{wrot}}$ is not proportional to $w\left(B_{\mathrm{a}}\right)$.

When $B \geq\left|B_{S}\right|$, however, $w(B)$ does not affect the scalar hysteretic property of $S_{2}$ (and $S_{1}$ ). Accordingly, $w(B)$ can be determined to directly control the rotational hysteresis loss for $B_{\mathrm{a}} \geq B_{\mathrm{S}}$. For example, when $n=1$, because $L_{\mathrm{rot}}\left(B_{\mathrm{a}}\right)$ becomes constant, $L_{\mathrm{wrot}}\left(B_{\mathrm{a}}\right)$ becomes proportional to $w\left(B_{\mathrm{a}}\right)$ as

$$
L_{\text {wrot }}\left(B_{\mathrm{a}}\right)=w\left(B_{\mathrm{a}}\right) L_{\mathrm{rot}}\left(B_{\mathrm{S}}\right)=w\left(B_{\mathrm{a}}\right) \frac{L_{\mathrm{wrot}}\left(B_{\mathrm{S}}\right)}{w\left(B_{\mathrm{S}}\right)}\left(B_{\mathrm{a}} \geq B_{\mathrm{S}}\right)
$$

\section{VECTOR HySTERETIC PROPERTY}

Two types of $w(B)$ below are examined for $|B|<B_{\mathrm{S}}$ :

i) a constant weighting function $w(B)=1$;

ii) the measured major loop width.

As shown in [12] and [13], the weighting function ii) improves the representation accuracy of the scalar stop model. For $|B| \geq B_{\mathrm{S}}$, two types of $w(B)$ below are compared:

A) a constant weighting function $w(B)=w\left(B_{\mathrm{S}}\right)$;

B) $w(B)=w\left(B_{\mathrm{S}}\right) B_{\mathrm{S}} /|B|$. 


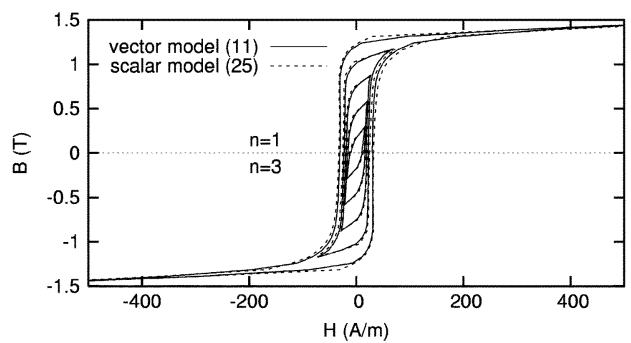

Fig. 4. Unidirectional hysteretic property.
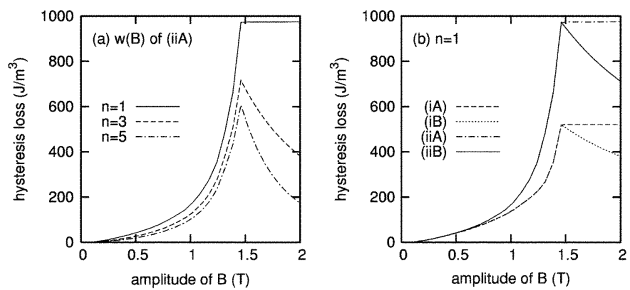

Fig. 5. Rotational hysteresis loss per cycle.
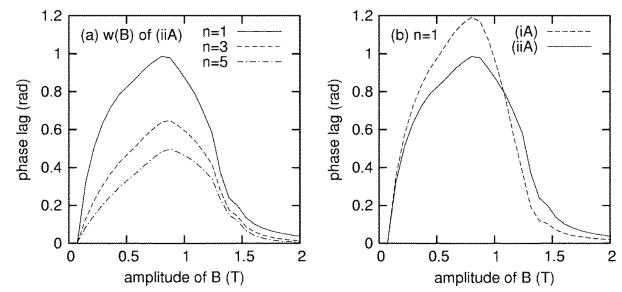

Fig. 6. Phase lag of $B$ to $H$.

Weighting function (B) is expected to reduce the rotational hysteresis loss with increased amplitude $B_{\mathrm{a}}$ when $B_{\mathrm{a}} \geq B_{\mathrm{S}}$. By combining the functions above, four types of weighting functions are obtained for examination: (iA), (iB), (iiA), and (iiB).

The dc magnetic property of a nonoriented silicon steel sheet (JIS: 50A290) is represented by the scalar and vector stop models. The magnetic property of the steel sheet along its rolling direction is used as the unidirectional property of an isotropic material although actual nonoriented silicon steel sheets do not have an isotropic property.

The scalar stop model (11) discretized with 20 hysterons is identified from 20 measured symmetric B-H loops. This scalar property is used to identify the vector stop model (25). Fig. 4 shows simulated BH loops that are obtained using the vector model $\boldsymbol{S}_{\mathrm{w}}(\boldsymbol{B})$ with $w(B)$ of (iiA) and $n=1,3$, where unidirectional characteristics are reconstructed accurately.

Next, the vector hysteretic property of $\boldsymbol{S}_{\mathrm{w}}(\boldsymbol{B})$ is examined for the rotational input of (13). Fig. 5(a) shows the rotational hysteresis loss yielded by $\boldsymbol{S}_{\mathrm{w}}(\boldsymbol{B})$ with $w(B)$ of (iiA) and $n=1,3,5$, whereas Fig. 5(b) is given by $\boldsymbol{S}_{\mathrm{w}}(\boldsymbol{B})$ with four types of $w(B)$ and $n=1$. Fig. 5(a) shows that the vector model with $n=1$ yields a constant hysteresis loss for $B \geq B_{\mathrm{S}}(=1.46 \mathrm{~T})$, which is not observed in actual ferromagnetic materials. The vector model with $n=3,5$ gives the hysteresis loss that decreases with the increase in $B_{\mathrm{a}}$ for $B_{\mathrm{a}} \geq B_{\mathrm{S}}$. Fig. 5(b) shows that the weighting function greatly affects the rotational loss. The rotational property also depends on $B_{\mathrm{S}}$. This examination uses $B_{\mathrm{S}}$ of $1.46 \mathrm{~T}$ as an assumed value for simulation; it is not an actual one for the silicon steel sheet. Fig. 6 shows the phase lag of $B$ to $H$, where the phase lag decreases with the increase in $n$. Measured data of rotational hysteretic property are required to determine $B_{\mathrm{S}}, n$ and $w(B)$.
The hysteresis loss for a unidirectional alternating input is unaffected by $n$ and $w(B)$ because the vector model is identified to reconstruct the unidirectional property as in Section III-D.

\section{CONCLUSION}

This paper generalizes a 2-D isotropic vector hysteresis model represented by the superposition of scalar stop models to improve the rotational property of the vector model. Based on an integral equation, an identification method of the generalized vector stop model is proposed.

The vector model reconstructs unidirectional hysteretic characteristics accurately. A projection parameter and a weighting function can control the rotational hysteresis loss of the vector model, which becomes proportional to the weighting function under saturation.

Comparison with measured rotational hysteretic characteristics of magnetic materials is required to confirm the representation capability of the vector model.

\section{ACKNOWLEDGMENT}

This work was supported by the Japan Society for the Promotion of Science, Grant-in-Aid for Scientific Research (C), 16560243.

\section{REFERENCES}

[1] I. D. Mayergoyz, Mathematical Models of Hysteresis and their Applications. Amsterdam, The Netherlands: Springer-Verlag, 2003.

[2] E. D. Torre, Magnetic Hysteresis. New York: IEEE Press, 1999.

[3] C. Stoner and E. P. Wohlfarth, "A mechanism of magnetic hysteresis in heterogeneous alloys," Phil. Trans. R. Soc., vol. 240A, pp. 599-642, 1948.

[4] H. Shimoji, M. Enokizono, T. Todaka, and T. Honda, "A new modeling of the vector magnetic property," IEEE Trans. Magn., vol. 38, no. 2, pt. 1, pp. 861-864, Mar. 2002.

[5] N. Takahashi, S. Miyabara, and K. Fujiwara, "Problems in practical finite element analysis using Preisach hysteresis model," IEEE Trans. Magn., vol. 35, no. 3, pp. 1243-1246, May 1999.

[6] M. A. Krasnosel'skii and A. V. Pokrovskii, Systems with Hysteresis. Berlin, Germany: Springer-Verlag, 1989.

[7] A. Visintin, Differential Models of Hysteresis. Berlin, Germany: Springer-Verlag, 1994.

[8] S. Bobbio, G. Miano, C. Serpico, and C. Visone, "Models of magnetic hysteresis based on play and stop hysterons," IEEE Trans. Magn., vol. 33, no. 6, pp. 4417-4426, Nov. 1997.

[9] J. V. Leite, N. Sadowski, P. K. Peng, and J. P. A. Bastos, "A new anisotropic vector hysteresis model based on stop hysterons," IEEE Trans. Magn., vol. 41, no. 5, pp. 1500-1503, May 2005.

[10] T. Matsuo, Y. Osaka, and M. Shimasaki, "Eddy-current analysis using vector hysteresis models with play and stop hysterons," IEEE Trans. Magn., vol. 36, no. 4, pt. 1, pp. 1172-1177, Jul. 2000, and its correction, vol. 36, no. 6, pp. 4050-4051, Nov. 2000.

[11] T. Matsuo and M. Shimasaki, "Isotropic vector hysteresis represented by superposition of stop hysteron models," IEEE Trans. Magn., vol. 37, no. 5, pt. 1, pp. 3357-3360, Sep. 2001, and its correction, vol. 37, no. 6, pt. 1, p. 3995, Nov. 2001.

[12] T. Matsuo, Y. Terada, and M. Shimasaki, "Stop model with input-dependent shape function and its identification methods," IEEE Trans. Magn., vol. 40, no. 4, pp. 1776-1783, Jul. 2004.

[13] T. Matsuo and M. Shimasaki, "Representation theorems for stop and play models with input-dependent shape functions," IEEE Trans. Magn., vol. 41, no. 5, pp. 1548-1551, May 2005.

[14] T. Matsuo and M. Shimasaki, "A method for optimal identification of a stop model with input-dependent shape function," IEEE Trans. Magn., vol. 42, no. 12, pp. 3818-3824, Dec. 2006. 